(1)

CrossMark

\title{
Oscillometry: old physiology with a bright future
}

\author{
Peter M.A. Calverley ${ }^{1}$ and Ramon Farré $2,3,4$
}

Affiliations: ${ }^{1}$ Institute of Ageing and Chronic Disease, University of Liverpool, Liverpool, UK. ${ }^{2}$ Unitat de Biofísica i Bioenginyeria, Facultat de Medicina i Ciències de la Salut, Universitat de Barcelona, Barcelona, Spain. ${ }^{3}$ CIBER de Enfermedades Respiratorias, Madrid, Spain. ${ }^{4}$ Institut d'Investigacions Biomediques August Pi Sunyer, Barcelona, Spain.

Correspondence: Peter M.A. Calverley, Institute of Ageing and Chronic Disease, University of Liverpool, William Henry Duncan Building, 6 West Derby Street, Liverpool, L7 8TX, UK. E-mail: pmacalaliverpool.ac.uk

@ERSpublications

Oscillometry is an old physiologically based technique that has recently been shown to be potentially useful for patient monitoring and personalised treatment. https://bit.ly/3e $4 \mathrm{j} 8 \mathrm{aA}$

Cite this article as: Calverley PMA, Farré R. Oscillometry: old physiology with a bright future. Eur Respir J 2020; 56: 2001815 [https://doi.org/10.1183/13993003.01815-2020].

Taking an ancient notion from Roman and Medieval authors [1], Isaac Newton wrote to Robert Hooke: 'if I have seen further, it is by standing on the shoulders of giants' [2]. This reasoning feels particularly true in the field of respiratory physiology. In 1956, Arthur DuBois helped describe a new method of measuring the lung volume of a person in a sealed chamber by measuring the pressure changes at the mouth and within the container [3]. Body plethysmography was born and is now used across the world in the diagnosis and management of many respiratory conditions. In the same year, DuBois and colleagues analysed the mechanical properties of the respiratory system during tidal breathing when an oscillating pressure was applied at high frequencies [4], and in 1968, Grimby and colleagues suggested that airway resistance in patients with obstructive lung diseases depends on the oscillation frequency [5]. This respiratory mechanics approach, which we reviewed in a previous editorial [6], is now called oscillometry, although many will know it better as the forced oscillation technique. It is much less well known than plethysmography and has been slower to make its way into clinical practice. Why is this?

Several elements have combined to make this a relatively neglected approach. One important factor could be called "intellectual inaccessibility". The principles underlying plethysmography are familiar to anyone who studied physics at school. By contrast, oscillometry translates signals from a time to a frequency domain using a fast Fourier transformation and generates negative numbers for values of reactance, which are often referred to as imaginary [7]. While this idea holds no terror for bioengineers, it is off-putting for less numerate physicians. A second issue has been largely technical. As pioneer investigators constructed their own oscillometric systems and data analysis was carried out manually (figure 1), original studies were time-consuming. The advent of modern computing, along with pressure transducers that accurately track pressure changes at high frequency, allowed commercial systems to be developed, although as noted previously [6], there is a lack of standardisation between equipment that can lead to different answers in the same individual [8]. The interpretation of oscillometric data requires the physician to take a different perspective from many other lung mechanics tests, which report the maximal ability of the lung to change volume or generate flow. Oscillometric measurements are made during quiet breathing and the results can be influenced by the end-expiratory lung volume at which data are collected. The resulting information is complementary to that from more familiar tests using forced expiratory manoeuvres. Oscillometry bears an approximate but not exact relationship with forced expiratory volume in $1 \mathrm{~s}$, which has frustrated those 
a)

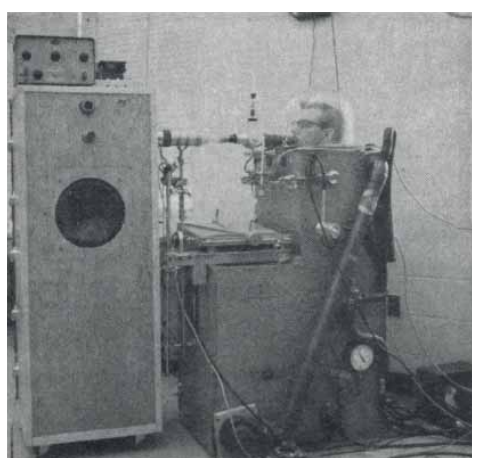

b)

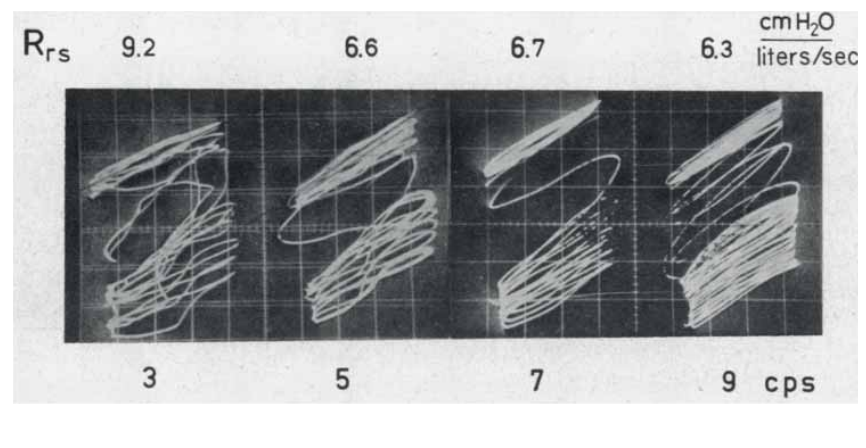

FIGURE 1 Example of a) the complex original experimental setting and b) the pressure and signals captured in an oscilloscope screen to be processed by hand in a seminal oscillometry study. Reproduced and modified from [5] with permission.

who wanted to use it to replace these routine tests. However, oscillometry does give us information about the way patients breathe in the face of mechanical abnormalities, and offers insight into how loads are distributed within the lungs.

Substantial progress has been made to reduce these barriers to uptake since our last editorial. In fact, the European Respiratory Society (ERS) recently published technical standards for oscillometry measurement in the European Respiratory Journal (ERJ) [9]. Documents like this are never a riveting read, even for an enthusiast, but they are vital if comparable information is to be collected in different laboratories using different equipment. There is now clear guidance about the approach to calibrating oscillometric systems and the way in which testing should be conducted. An important requirement is the provision of data about the characteristics of the system used when reporting research findings, which will help us interpret apparent differences between centres as well as stimulating good clinical practice in the routine laboratory.

The clinical applications of oscillometry are wide. The effort-independent nature of the measurement and its simplicity from the patient's perspective have led to its early adoption in paediatric practice, where it provides objective lung function information in settings in which more complex testing would be impossible or hard to reproduce. Similarly, there is likely to be a role for this technique in neonatal/ paediatric [10] and adult intensive care units, for instance to monitor COPD ventilated patients [11], to assess the effect of end-expiratory pressure [12] or to assess weaning from mechanical ventilation [13]. Moreover, oscillometry has been shown to be very useful for monitoring how airway resistance is normalised by application of continuous positive airway pressure (CPAP) therapy in patients with obstructive sleep apnoea (OSA) [14]. In fact, the technique has been incorporated into commercial CPAP devices, both for titrating the prescribed nasal pressure [15] and to continuously adapting nasal pressure to each OSA patient through the night [16]. In conscious adults, the main application of oscillometry lies in the field of airways disease. Important variables in adults are the resistance and reactance at relatively low frequencies, e.g. $5 \mathrm{~Hz}$ (now separated into inspiratory and expiratory values), as well as data about the resistance at higher frequencies, e.g. $20 \mathrm{~Hz}$, together with the frequency dependence of resistance (for example, the resistance reduction between $5 \mathrm{~Hz}$ and $20 \mathrm{~Hz}$ ), which reflects the resistive properties of the periphery of the lung and chest wall as well as the degree of mechanical heterogeneity within the lungs. This variable has been shown to define discrete phenotypes among asthmatics [17]. Although initial data in stable COPD was rather disappointing [18], the recognition that tidal expiratory flow limitation (EFL) could be identified by looking at the difference between inspiratory and expiratory reactance values allowed for a better explanation of the effects of bronchodilators in COPD [19]. COPD patients with tidal EFL are more breathless and more likely to exacerbate than patients without tidal EFL [20, 21]. Respiratory system reactance and inspiratory resistance change as COPD patients recover from exacerbations [22], which has led workers in Sydney (Australia) to try to identify what happens to tidal breathing lung mechanics as exacerbations develop.

In this issue of the ERJ, ZIMMERMANn et al. [23] report a study of 15 moderately severe symptomatic COPD patients followed over 8-9 months with daily oscillometry recordings at home. The authors looked at the day-to-day variation in $5 \mathrm{~Hz}$ resistance and reactance, focussing on the inspiratory values, the standard deviations of inspiratory reactance $\left(\mathrm{SDX}_{\mathrm{insp}}\right)$ and of inspiratory resistance $\left(\mathrm{SDR}_{\mathrm{insp}}\right)$. Data were recorded as a 7-day running average value, although other time periods were considered. They found a clear relationship between day-to-day variation in reactance and COPD assessment test (CAT) scores, a measure 
of individual health status. CAT score worsened as an exacerbation developed and so did SDX insp $_{\text {, which }}$ could reliably detect an impending exacerbation before the CAT score changed, especially when the running average time window was decreased to 5 rather than 7 days.

Several important points emerge from this report. First, home monitoring with oscillometry is possible with high degrees of adherence and good data quality, as previously reported by WALKeR et al. [24] in a much larger pan-European study using the same equipment. Second, day-to-day changes in reactance identify more symptomatic people and have the potential to identify exacerbations needing medical therapy early in their natural history. Here, the change in inspiratory reactance rather than resistance was the important signal. Multiple explanations are possible for this finding, including an increase in the magnitude of mechanical heterogeneity within the lungs and/or day-to-day fluctuations in airway mechanics. Further research will be needed to determine which mechanisms are relevant in which patients. Perhaps most important is the observation that $\mathrm{SDX}_{\text {insp }}$ variation was highly predictive of exacerbations in some patients but not useful in others. This emphasises the heterogeneity of COPD exacerbations and the fact that not all episodes that lead to symptoms are likely to be accompanied by significant changes in lung mechanics. Further data in larger datasets are needed to explore this issue further.

Just as in sleep, where CPAP pressures can be automatically adjusted to maintain upper airway patency using oscillometry [25], so it is interesting to speculate what would happen if extrinsically applied positive end-expiratory pressure (PEEP) was adjusted to overcome tidal EFL in conscious patients with stable COPD. This concept has been explored by SuH et al. [26] in an elegant physiological study also published in this issue of the ERJ. Tidal EFL is more common in patients when supine, which helps explain why only eight of their 10 severe COPD patients showed this on testing when erect. The patients who were already using home noninvasive ventilation had their transdiaphragmatic pressure swings recorded, along with their neural respiratory drive assessed by electrodes placed over their parasternal muscles. The authors used a novel ventilator system, which adjusted extrinsic PEEP until tidal EFL, as determined by the changes in reactance between inspiration and expiration, returned to normal, an approach suggested by earlier work in similarly instrumented COPD patients [27]. By adjusting the applied extrinsic pressure above and below the "optimal" value that abolished tidal EFL, they found that when flow limitation was removed, the patients had less intrinsic PEEP, a recognised load on the respiratory muscles, smaller transdiaphragmatic pressure swings and a lower neural drive to breathe. Identifying this physiological optimal condition would not have been possible without oscillometry measurements. It is no surprise in these heavily instrumented patients that there were no consistent changes in reported levels of breathlessness, even when neural drive was lower, and more data will be needed to be sure that this physiological benefit translates into improvements in ventilator adherence. Nonetheless, this is a promising and novel approach to a more personalised ventilator strategy in COPD.

These new studies $[23,26]$, although small, offer a glimpse into the possibilities that oscillometry promises not just as a diagnostic modality but also in effort-independent patient monitoring and even therapy. In our editorial 7 years ago, we concluded with the hope that oscillometry would improve clinical diagnosis and our disease understanding [6]. Those hopes have been amply realised. As clinicians grow more familiar with these applications and less intimidated by the terminology, it seems likely that oscillometry will become part of our routine medical practice. Hopefully, more use in patients will trigger the interest of industry to produce more simple and affordable devices, in a virtuous circle leading to extended application of oscillometry in the clinical arena.

Conflict of interest: P.M.A. Calverley has nothing to disclose. R. Farre has nothing to disclose.

\section{References}

$1 \quad$ Fakes and forgeries in the Middle Ages. In: Eco U. From the Tree to the Labyrinth: Historical Studies on the Sign and Interpretation. Cambridge, Harvard University Press, 2014; pp. 223-249.

2 Newton I. Letter to Robert Hooke, February 5, 1675. https://digitallibrary.hsp.org/index.php/Detail/objects/9792

3 DuBois AB, Botelho SY, Bedell GN, et al. A rapid plethysmographic method for measuring thoracic gas volume: a comparison with a nitrogen washout method for measuring functional residual capacity in normal subjects. J Clin Invest 1956; 35: 322-326.

4 DuBois AB, Brody AW, Lewis DH, et al. Oscillation mechanics of lungs and chest in man. J Appl Physiol 1956; 8: 587-594.

5 Grimby G, Takishima T, Graham W, et al. Frequency dependence of flow resistance in patients with obstructive lung disease. J Clin Invest 1968; 47: 1455-1465.

6 Calverley PM, Farre R. Putting noninvasive lung mechanics into context. Eur Respir J 2013; 42: 1435-1437.

7 Bates JH, Irvin CG, Farré R, et al. Oscillation mechanics of the respiratory system. Compr Physiol 2011; 1: $1233-1272$. 
8 Dandurand RJ, Lavoie JP, Lands LC, et al. Comparison of oscillometry devices using active mechanical test loads. ERJ Open Res 2019; 5: 00160-2019.

9 King GG, Bates J, Berger KI, et al. Technical standards for respiratory oscillometry. Eur Respir J 2020; 55: 1900753.

10 Hantos Z, van Kaam AH, McEvoy CT, et al. An official American Thoracic Society/European Respiratory Society workshop report: Evaluation of respiratory mechanics and function in the pediatric and neonatal intensive care units. Ann Am Thorac Soc 2016; 13: S1-S11.

11 Farré R, Ferrer M, Rotger M, et al. Respiratory mechanics in ventilated COPD patients: forced oscillation versus occlusion techniques. Eur Respir J 1998; 12: 170-176.

12 Kaczka DW, Ingenito EP, Body SC, et al. Inspiratory lung impedance in COPD: effects of PEEP and immediate impact of lung volume reduction surgery. J Appl Physiol 2001; 90: 1833-1841.

13 Sellares J, Acerbi I, Loureiro $\mathrm{H}$, et al. Respiratory impedance during weaning from mechanical ventilation in a mixed population of critically ill patients. Br J Anaesth 2009; 103: 828-832.

14 Navajas D, Farre R, Rotger M, et al. Assessment of airflow obstruction during CPAP by means of forced oscillation in patients with sleep apnea. Am J Respir Crit Care Med 1998; 157: 1526-1530.

15 Galetke W, Randerath WJ, Stieglitz S, et al. Comparison of manual titration and automatic titration based on forced oscillation technique, flow and snoring in obstructive sleep apnea. Sleep Med 2009; 10: 337-343.

16 Herkenrath SD, Treml M, Anduleit N, et al. Extended evaluation of the efficacy of a proactive forced oscillation technique-based auto-CPAP algorithm. Sleep Breath 2019; 24: 825-833

17 Postma DS, Brightling C, Baldi S, et al. Exploring the relevance and extent of small airways dysfunction in asthma (ATLANTIS): baseline data from a prospective cohort study. Lancet Respir Med 2019; 7: 402-416.

18 Crim C, Celli B, Edwards LD, et al. Respiratory system impedance with impulse oscillometry in healthy and COPD subjects: ECLIPSE baseline results. Respir Med 2011; 105: 1069-1078.

19 Dellacà RL, Pompilio PP, Walker PP, et al. Effect of bronchodilation on expiratory flow limitation and resting lung mechanics in COPD. Eur Respir J 2009; 33: 1329-1337.

20 Aarli BB, Calverley PM, Jensen RL, et al. Variability of within-breath reactance in COPD patients and its association with dyspnoea. Eur Respir J 2015; 45: 625-634.

21 Aarli BB, Calverley PM, Jensen RL, et al. The association of tidal EFL with exercise performance, exacerbations, and death in COPD. Int J Chron Obstruct Pulmon Dis 2017; 12: 2179-2188.

22 Stevenson NJ, Walker PP, Costello RW, et al. Lung mechanics and dyspnea during exacerbations of chronic obstructive pulmonary disease. Am J Respir Crit Care Med 2005; 172: 1510-1516.

23 Zimmermann SC, Huvanandana J, Nguyen CD, et al. Day-to-day variability of forced oscillatory mechanics for early detection of acute exacerbations in COPD. Eur Respir J 2020; 56: 1901739.

24 Walker PP, Pompilio PP, Zanaboni $\mathrm{P}$, et al. Telemonitoring in chronic obstructive pulmonary disease (CHROMED). A randomized clinical trial. Am J Respir Crit Care Med 2018; 198: 620-628.

25 Badia JR, Farré RO, John Kimoff R, et al. Clinical application of the forced oscillation technique for CPAP titration in the sleep apnea/hypopnea syndrome. Am J Respir Crit Care Med 1999; 160: 1550-1554.

26 Suh E-S, Pompilio P, Mandal S, et al. Autotitrating external positive end-expiratory airway pressure to abolish expiratory flow limitation during tidal breathing in patients with severe COPD: a physiological study. Eur Respir J 2020; 56: 1902234

27 Zannin E, Chakrabarti B, Govoni L, et al. Detection of expiratory flow limitation by forced oscillations during noninvasive ventilation. Am J Respir Crit Care Med 2019; 200: 1063-1065. 